\title{
A Physical Channel Model and Analysis for Nanoscale Molecular Communications with Förster Resonance Energy Transfer (FRET)
}

\author{
Murat Kuscu, Student Member, IEEE and Ozgur B. Akan, Senior Member, IEEE
}

\begin{abstract}
In this study, a novel and physically realizable nanoscale communication paradigm is introduced based on a well-known phenomenon, Förster Resonance Energy Transfer (FRET) for the first time in the literature. FRET is a nonradiative energy transfer process between fluorescent molecules based on the dipole-dipole interactions of molecules. Energy is transferred rapidly from a donor to an acceptor molecule in a close proximity such as 0 to $10 \mathrm{~nm}$ without radiation of a photon. Low dependency on the environmental factors, controllability of its parameters and relatively wide transfer range make FRET a promising candidate to be used for a high rate nanoscale communication channel. In this paper, the simplest form of the FRET-based molecular communication channel comprising a single transmitter-receiver nanomachine pair and an extended version of this channel with a relay nanomachine for long range applications are modeled considering nanomachines as nanoscale electromechanical devices with some sensing, computing and actuating capabilities. Furthermore, using the information theoretical approach, the capacities of these communication channels are investigated and the dependency of the capacity on some environmental and intrinsic parameters is analyzed. It is shown that the capacity can be increased by appropriately selecting the donor-acceptor pair, the medium, the intermolecular distance and the orientation of the molecules.
\end{abstract}

Index Terms-FRET, nanoscale communications.

\section{INTRODUCTION}

$\mathbf{N}$ ANOSCALE communication is a novel and quite interdisciplinary research area. Several potential approaches have been proposed in order to achieve communication in the nanoscale such as electromagnetic, acoustic or molecular [1][3]. In this paper, we introduce a novel and radically different method for the communication in the nanoscale by exploiting a well-known, physical controllable phenomenon, Förster (or Fluorescence) Resonance Energy Transfer (FRET).

FRET is a non-radiative energy transfer process between fluorescent molecules and it is widely used in studies of biotechnological research including fluorescence microscopy, molecular biology and optical imaging [4], [5]. The phenomenon yields a significant amount of structural information about the donor and acceptor pair, therefore, many methods based on FRET have been developed and used in these areas. For example, using its strong dependence on distance, FRET

The authors are with the Next-generation and Wireless Communications Laboratory, Department of Electrical and Electronics Engineering, Koc University, Istanbul, 34450, Turkey (e-mail: mkuscu, akan@ku.edu.tr).

This work was supported in part by the Turkish Scientific and Technical Research Council under grant \#109E257, by the Turkish National Academy of Sciences Distinguished Young Scientist Award Program (TUBA-GEBIP), and by IBM through IBM Faculty Award. is exploited as a spectroscopic ruler [6] while determining the intramolecular and intermolecular distances and monitoring the conformational changes of proteins [7]. FRET is also used in molecular and quantum computing studies as a tool for creation of entangled quantum states [8], [9].

In this study, for the first time in the literature, FRET has been approached from the communication perspective and introduced as a novel molecular communication paradigm. There are many biologically inspired and theoretically modeled molecular communication techniques in the literature including the communication models devised based on intercellular calcium signaling [10], pheromones [11], flagellated bacteria and catalytic nanomotors [12], carbon nanotubes [13], pollen and spores [11], as well as morphogenesis [14] in order to encode, transfer and decode information. FRETbased communication method is based on a physically existing phenomenon and unlike the other techniques it provides significantly higher capacity communication. The excited state energy of molecules that conveys the information is transferred in the nanosecond range so that FRET-based communication is incomparably faster than the already proposed nanoscale communication techniques. Furthermore, high-level controllability of almost all of the system parameters makes FRET-based channel more reliable. The abundance of both theoretical and practical studies about FRET in the literature and availability of its experimental setups provide the opportunity of making improvements validating theoretical model based on experiments. Hence, unlike most of the existing approaches in the literature, we introduce an already analyzed and experimented, therefore, a physically realizable, and hence, clearly realistic solution to the problem of nanoscale communication.

In this paper, we have also extended our work in [15] by enhancing the FRET-based communication channel in order to realize nanoscale communication over distances longer than $10 \mathrm{~nm}$ with the integration of a relay node between transmitter and receiver. In the extended model, excited energy of the donor is transferred first to the fluorophore on the relay nanomachine, afterwards the excited relay transfers its energy to the acceptor on the receiver via FRET. This sequential energy transfer (also called multi-step FRET in the literature) is achieved experimentally in some studies related to fluorescence spectroscopy such as [16], [17] locating several numbers of fluorophores in a linear order over several distances. This enhancement shows the potential of the FRET-based nanoscale communication in the sense that it can be extended further being a solution for long range nanoscale communication and 
similar network schemes such as multiple-access or broadcast.

The remainder of this paper is organized as follows. In Section II of the study, we explain the basic concepts of FRET and underline the governing physical laws and its mathematical formulation. In section III, we model the FRETbased communication channel with a single donor-acceptor pair. In section IV, the extended version of the channel with an addition of a relay node is modeled to realize a longer range communication based on FRET. An information theoretical analysis of FRET-based channel is performed in Section $\mathrm{V}$ in order to determine the closed-form expression for the capacity of the channel. In Section VI, we analyze the dependence of the channel capacity on some environmental and intrinsic factors and demonstrate a selection strategy for these parameters to achieve higher communication capacity. Besides, we investigate the dependence of the capacity of the channel with a relay node on internodal distances. Finally, the concluding remarks are given in Section VII.

\section{THEORY OF FRET}

FRET is non-radiative energy transfer process observed among fluorescent molecules (fluorophores), i.e., the molecules with the ability of absorbing energy at a specific range of wavelength and emitting that absorbed energy at a different wavelength [18]. Quantum dots, organic dyes and polymers are the most frequently used fluorophores in FRET studies. This electrodynamic phenomenon is based on longrange dipole-dipole interaction between a donor fluorophore in its excited state and an acceptor fluorophore in its ground state which are in a close proximity. When the donor is excited by an incident light, it may transfer its energy to an acceptor molecule through FRET and relaxes to its ground state, if certain conditions are satisfied. First of all, donor and acceptor must be located in a close proximity like $0-10 \mathrm{~nm}$. In addition, the spectral characteristics of donor and acceptor must have significant similarity, i.e., the overlapping area of the emission spectrum of donor and the absorption spectrum of acceptor, must be large enough. If these conditions are not satisfied, FRET does not occur and in the case of donor excitation, donor relaxes to its ground state by releasing a photon.

In 1948, Theodor Förster postulated the governing equations in the theory of non-radiative energy transfer in his wellknown article [19]. The first parameter that characterizes the donor-acceptor pairs is the Förster Radius, the distance between donor and acceptor when the efficiency of the energy transfer is $50 \%$ and given by

$$
R_{0}^{6}=\frac{9000 \ln (10) \kappa^{2} Q_{D}}{128 \pi^{5} N n^{4}} \int_{0}^{\infty} F_{D}(\lambda) \epsilon_{A}(\lambda) \lambda^{4} \mathrm{~d} \lambda
$$

where the $\kappa^{2}$ is the orientation factor, $Q_{D}$ is the quantum yield of the donor, $n$ is the refractive index of the medium, $N$ is the Avagadro's number. The integral part of (1) yields the degree of the overlap of the emission spectrum of the donor and the absorption spectrum of the acceptor and denoted by $J(\lambda)$,

$$
J(\lambda)=\int_{0}^{\infty} F_{D}(\lambda) \epsilon_{A}(\lambda) \lambda^{4} \mathrm{~d} \lambda
$$

where $F_{D}(\lambda)$ is the normalized fluorescence emission intensity and $\epsilon_{A}(\lambda)$ is the acceptor molar absorptivity. Here, the orientation factor $\left(\kappa^{2}\right)$ is the most problematic, i.e., with the highest uncertainty, parameter given in [20] as

$$
\kappa^{2}=\left(\cos \theta_{T}-3 \cos \theta_{D} \cos \theta_{A}\right)^{2}
$$

where $\theta_{T}, \theta_{D}$ and $\theta_{A}$ are the angles determined by the emission and absorption transition dipoles of the fluorophores as shown in Fig. 1. It is not possible to determine the exact relative orientation of donor and acceptor, i.e., the exact value of $\kappa^{2}$, however, it ranges between 0 and 4 . In most studies, donor and acceptor are supposed to randomly rotate and $\kappa^{2}$ is assumed to be $2 / 3$ which is the mean value [18].

The efficiency of energy transfer as a function of intermolecular distance and Förster radius, $R_{0}$ is formulated as

$$
E(R)=\frac{R_{0}^{6}}{R_{0}^{6}+R^{6}}
$$

where $R$ is the distance between donor and acceptor [18].

The rate of the energy transfer is given by

$$
k_{T}(R)=\frac{1}{\tau_{D}}\left(\frac{R_{0}}{R}\right)^{6}
$$

where $\tau_{D}$ is the donor lifetime, i.e., the average time the donor remains in its excited state [18]. Normally, in the absence of any non-radiative relaxation process, it is determined by only the radiative emission rate, $k_{R}$ and given in [18] as

$$
\tau_{D}=\frac{1}{k_{R}}
$$

In the case of FRET, the energy transfer to the acceptor causes a reduction in the donor's excited state lifetime and it can be formulated as

$$
\tau_{D A}=\frac{1}{k_{R}+k_{T}}
$$

where $\tau_{D A}$ is the donor's excited state lifetime before it releases the energy through FRET [18].

\section{FRET-BASEd NANoscale Communication CHANNEL}

We constructed our communication model with a single donor fluorescence molecule (D) bound to a transmitter nanomachine (TN), and a single acceptor fluorescence molecule (A) bound to a receiver nanomachine (RN), at fixed locations separated by a reasonable distance $R$ in an aqueous medium considering FRET as the communication channel with the system exciton, i.e., the combined state of electron and hole, being a carrier as shown in Fig. 1, where $\theta_{T}$ is the angle between the emission transition dipole of donor and the absorption transition dipole of the acceptor, $\theta_{D}$ and $\theta_{A}$ are the angles between these dipoles and vector joining the donor and the acceptor, respectively. The arrow between (D) and (A) shows the FRET direction. Other arrows show the transition dipoles of (D) and (A) as well as the direction of incoming photon with a wavelength of $\lambda_{i}$. Assuming the molecules are properly selected, i.e., they have sufficient spectral overlap $(J(\lambda))$, in the case of a donor excitation, FRET occurs with probability of $P_{F R E T}$. 


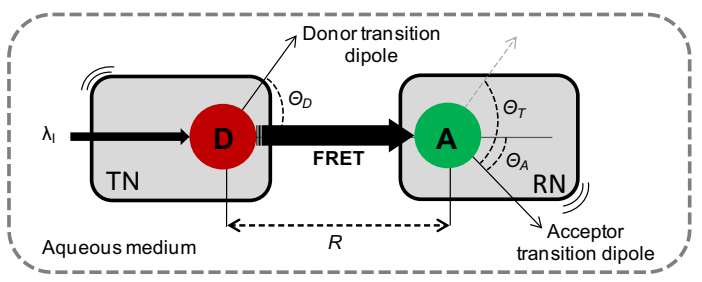

Fig. 1. Point-to-point FRET-based molecular communication channel model with single TN and RN communicating via FRET.

When a laser source excites the donor at a proper wavelength, basically there are two ways of relaxation for the donor, the first one is through radiative emission, i.e., fluorescence and the second one is through FRET disregarding the other low-probability non-radiative relaxation pathways such as dissipation as heat or collision with another molecule, i.e., collisional quenching as well as intersystem crossing to an excited triplet state which can actually result in phosphorescence.

In FRET spectroscopy, the FRET efficiency is determined by continuously exciting the donor and calculating the proportion of the number of FRET relaxations to the number of total relaxation processes in a specified time interval [21]. Therefore, for a single cycle of excitation and relaxation of the donor, the efficiency can be considered as the probability of the excited donor to relax via FRET. Thus, using (4), for a single exciton, the probability of FRET as a function of intermolecular distance can be given by

$$
P_{F R E T}(R)=E(R)=\frac{R_{0}^{6}}{R_{0}^{6}+R^{6}}
$$

where $R_{0}$ is the Förster radius and it is calculated according to (1). In [22], in a medium of water with a refractive index of 1.3342 at $25^{\circ} \mathrm{C}$, assuming rapid randomization of relative orientation of molecules, i.e., $\kappa^{2}=2 / 3$, for a pair consisting of ECFP (Enhanced Cyan Fluorescent Protein) as donor and EYFP (Enhanced Yellow Fluorescent Protein) as acceptor, the Förster radius is calculated as $4.92 \mathrm{~nm}$. Both ECFP and EYFP are the variants of Green Fluorescent Protein (GFP) and widely used in various fluorescence spectroscopy applications for their similar spectral characteristics, photostability, high extinction coefficients and high quantum yields [23]. At the same time, the pair ECFP-EYFP is a good candidate for communication purposes due to the relatively large value of $R_{0}$.

In FRET-based communication model, donor excitation is realized by a pulsed laser which has a waveshape approximated in Fig. 2. The duration of pulses can be selected very short compared to the lifetimes of the fluorophores used because of energy saving concern. The laser is considered as the main information source of the communication system and is not considered to be a part of $\mathrm{TN}$ at present due to energy and size limitations. Vast number of photons released by the laser with wavelengths near to the excitation maximum of the donor, guarantee the donor excitation in femtoseconds duration, since the absorption probability of the donor is almost 1 for that wavelengths. Therefore, in the subsequent probability calculations, the probability of donor excitation at any instant of laser pulse duration is assumed to be 1 .

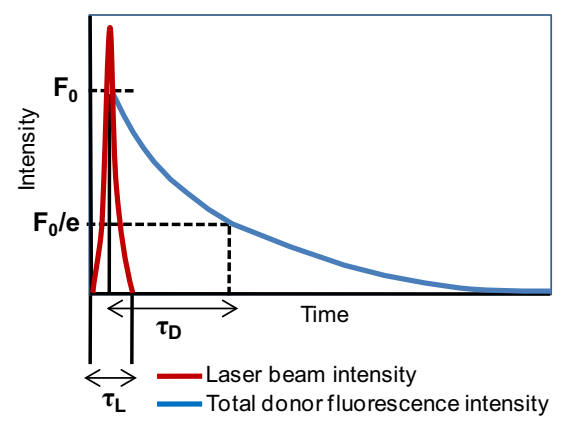

Fig. 2. Approximated laser pulse shape and lifetime determination.

Excited state lifetime is determined by measuring and recording the fluorescence times of many fluorophores of the same kind after an excitation by a very small duration pulsed laser and calculating the mean of these records as shown in Fig. 2. Therefore, it is very possible for the employed donor and acceptor not to have the anticipated lifetimes. The lifetime of a fluorophore is a critical parameter in determination of the laser excitation period $\left(T_{H}\right)$ in the sense that an excited fluorophore cannot be re-excited until it relaxes to the ground state [24]. For example, the donor molecule cannot transfer the excited state energy to the acceptor through FRET if the acceptor is still in its excited state as a result of the preceding FRET process. Therefore, we consider the worst -with the longest duration- case while determining $T_{H}$.

In the model, we implemented On-Off Keying Modulation with two bits available as in the traditional digital communications. The excitation of the donor by the information source at the beginning of a time interval $\left(T_{H}\right)$ corresponds to bit 1 , and no-excitation at the beginning of a time interval corresponds to bit 0 . The $\mathrm{RN}$ is assumed to have the ability of checking whether the acceptor is excited through FRET or not during the corresponding time interval. If it is excited through FRET, it decides that the TN transmitted bit 1, otherwise it decides that the $\mathrm{TN}$ transmitted bit 0 . With further investigations on nanoscale system design, such RN designs can be potentially realizable. At current FRET studies, acceptor fluorescence is observed with a system comprising a Photomultiplier Tube (PMT) and a Near-field Scanning Optical Microscope (NSOM) in order to detect whether or not FRET occurs [25]. This mechanism is considered to be used as the ultimate destination of information in the future studies aiming to validate the FRET-based communication channel experimentally.

In Fig. 3, conceptually we demonstrate the different cases for data transmission. For the case in Fig. 3-a, a laser directed to the donor releases a pulse which has a duration of $\tau_{L}$. The wavelength of photons released by laser is near to the excitation maximum of the donor in order to guarantee the donor excitation. During $\tau_{L}$, donor absorbs a photon and becomes excited. In the excited state, after an average time $\tau_{D A}$, i.e., the lifetime of donor in the case of FRET, donor succeeds in transferring the excited energy to the acceptor through FRET with the probability of $P_{F R E T}(R)$. Therefore, acceptor on RN also becomes excited through FRET. The time required for FRET to be completed is the reciprocal 


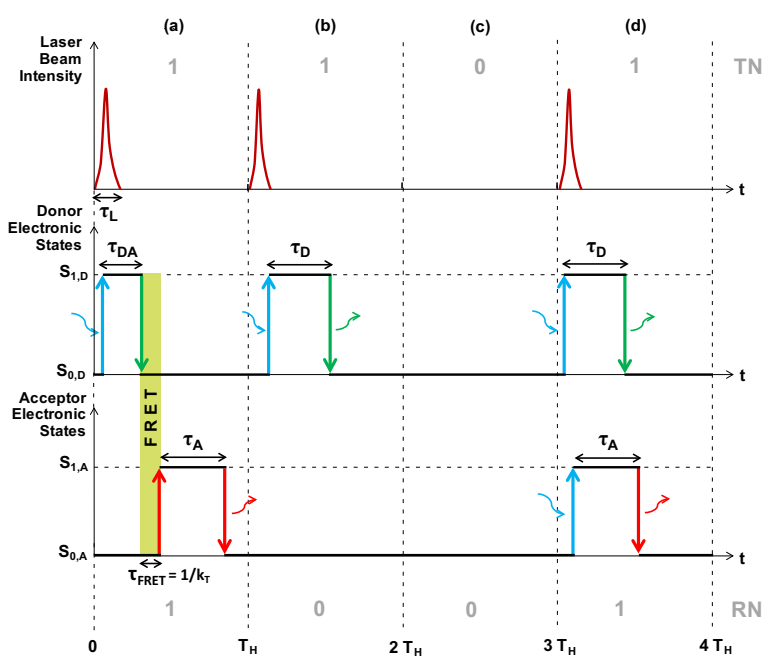

Fig. 3. Example data stream demonstrating pulsed laser beam intensity, donor and acceptor state transitions for different cases. (a) FRET case; bit 1 is transmitted by TN, bit 1 is detected by RN. (b) No-FRET case; bit 1 is transmitted, bit 0 is detected. (c) No-excitation case; bit 0 is transmitted, bit 0 is detected. (d) Direct excitation case; bit 1 is transmitted, bit 1 is detected.

of FRET rate, i.e., $\tau_{F R E T}(R)=1 / k_{T}(R)=\tau_{D}\left(R / R_{0}\right)^{6}$. Acceptor stays in excited state for an average time of $\tau_{A}$ and then it relaxes by emitting a photon. $\mathrm{RN}$ detects the excited state of acceptor in the time interval $0-T_{H}$ and decides bit 1 . In the figure, excitation and fluorescence durations are neglected, since they are too small compared to the lifetimes and FRET duration which are approximated according to the measured values for previously mentioned fluorescent protein pair ECFP-EYCP. The measured lifetimes are $2.68 \mathrm{~ns}$ for the donor ECFP and $2.88 n s$ for the acceptor EYFP [26].

In Fig. 3-b, again the donor is excited to transmit bit 1, however, this time donor fails to deliver its excited energy to the acceptor molecule of $\mathrm{RN}$ with the probability of $1-$ $P_{F R E T}(R)$ in the time interval $T_{H}-2 T_{H}$. Therefore, RN cannot detect an excited state during this time interval and decides bit 0 . The probability of failure for this case can be minimized by decreasing the intermolecular distance.

In Fig. 3-c, the laser source does not release pulse in order for $\mathrm{TN}$ to transmit bit 0 , therefore, the donor is not excited in the time interval $2 T_{H}-3 T_{H}$. As a result, the acceptor also is not excited and RN decides the correct bit, i.e., bit 0 .

In Fig. 3-d, the laser excites both the donor and the acceptor directly. This situation is known as direct excitation and can be problematic for FRET applications in fluorescence microscopy. Direct excitation occurs when donor is excited by a laser at a wavelength that belongs to excitation spectrum of both donor and acceptor. Although direct excitation can be minimized by using a laser with a small excitation volume and appropriately directing the laser source to the donor molecule in $\mathrm{TN}$, it is seen in Fig. 3-d that direct excitation does not result in any confusion for $\mathrm{RN}$ to detect the correct bit.

The case in Fig. 3-a can be extended to the worst -with the longest duration- case for data transmission to determine minimum excitation period, i.e., $T_{H-m i n}$. Since the fluorophores with lifetimes longer than the determined average lifetime constitute a small portion of the entire as seen in

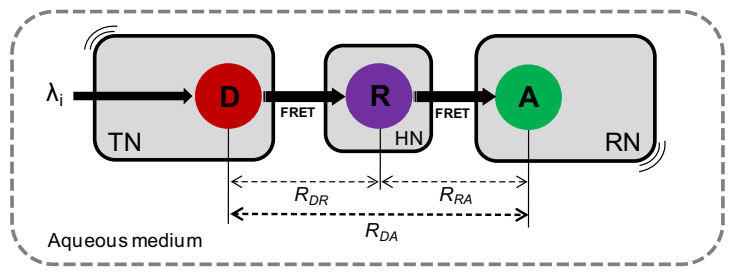

Fig. 4. Long-range FRET-based communication model with multi-step FRET.

Fig. 2, in order to ease the calculation, we assume that the maximum values of lifetimes are the same as the mean values, i.e., $\tau_{D-\max }=\tau_{D}$ and $\tau_{A-\max }=\tau_{A}$ as well as $\tau_{D A-\max }=\tau_{D A}$. Assuming that the donor excitation occurs with the absorption of the last photon released by laser and neglecting the excitation time of the donor and disregarding direct excitation, in order to prevent intersymbol interference, the minimum value of the excitation period $T_{H}$ is given as

$$
T_{H-\min }=\tau_{L}+\tau_{D A}+\tau_{F R E T}+\tau_{A}
$$

$T_{H}$ must be reasonably greater than the minimum value considering the weakness of the assumptions made.

\section{LONG-RANGE FRET-BASEd COMMUNiCATION CHANNEl With MUlTi-STEP FRET}

In the literature, there are several studies that achieve multistep FRET for transmission of excited energy over distances longer than $10 \mathrm{~nm}$ [16], [17]. Furthermore, in one of the related works, multi-step FRET is proposed for design of a photonic wire [27]. Multi-step FRET is realized by employing relay molecules between donor and acceptor. These relay nodes act like both donor and acceptor at the same time, i.e., they transfer the energy absorbed via FRET to the next groundstate molecule in close proximity through FRET. A long-range version of FRET-based communication comprising sequential FRET channels can be realized using multi-step method.

In Fig. 4, the simplest form of multi-step FRET-based communication model with one transmitter nanomachine (TN) with a donor fluorophore (D), one receiver nanomachine (RN) with an acceptor fluorophore (A) and one relay nanomachine $(\mathrm{HN})$ with a relay fluorophore $(\mathrm{R})$ is demonstrated. The arrows between (D), (R) and (A) show the FRET direction. Other arrow shows the direction of incoming photon with a wavelength of $\lambda_{i}$. The nanomachines are located linearly to improve the energy transfer range, therefore, the intermolecular distance between the donor fluorophore on TN and the acceptor fluorophore on RN, i.e., $R_{D A}$ is given by

$$
R_{D A}=R_{D R}+R_{R A}
$$

where $R_{D R}$ is the distance between the donor and relay and $R_{R A}$ is the distance between the relay and acceptor.

The overlap between the emission spectrum of donor and the absorption spectrum of the relay as well as the overlap between the emission spectrum of relay and the absorption spectrum of the acceptor must be significantly large to allow multi-step FRET to occur between these molecules. The probability of direct FRET between $\mathrm{TN}$ and $\mathrm{RN}$ is ignored assuming $R_{D A}$ is significantly greater than $R_{0, D A}$, i.e., the 


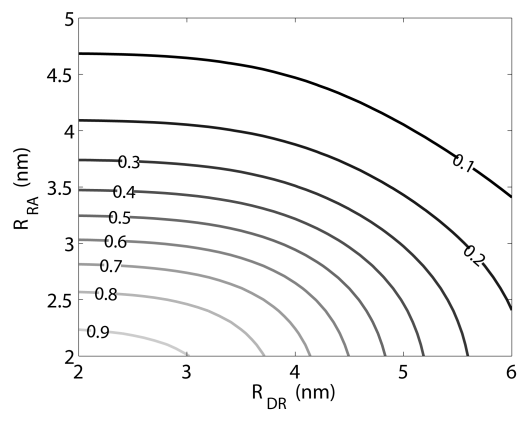

Fig. 5. $\quad P_{F R E T, D A}$ with varying $R_{D R}$ and $R_{R A}$ for $\operatorname{ECFP(D)-EYFP(R)-~}$ EGFP(A) arrangement.

Förster distance of donor-acceptor pair. As a consequence, the direct communication between $\mathrm{TN}$ and $\mathrm{RN}$ is assumed to be disabled. Therefore, the multi-step channel is considered as a series of two independent channels. Therefore, the overall transfer efficiency between the donor and acceptor via multistep FRET, i.e., $E_{D A}$ is given by

$$
E_{D A}=E_{D R} \times E_{R A}
$$

where $E_{D R}$ is the FRET efficiency between the donor and relay fluorophore and $E_{R A}$ is the FRET efficiency between the relay and acceptor fluorophore [17]. Using (4), (8) and (11), the probability of FRET between the donor and acceptor for a single exciton in terms of $R_{D R}$ and $R_{R A}$ is given by

$$
P_{F R E T, D A}\left(R_{D R}, R_{R A}\right)=\frac{R_{0, D R}^{6}}{R_{0, D R}^{6}+R_{D R}^{6}} \times \frac{R_{0, R A}^{6}}{R_{0, R A}^{6}+R_{R A}^{6}}
$$

where $R_{0, D R}$ is the Förster distance of donor-relay pair and $R_{0, R A}$ is the Förster distance of relay-acceptor pair. As it is seen in (12), if $R_{0, D R}$ and $R_{0, R A}$ are not equal, $P_{F R E T, D A}$ is not directly related to $R_{0, D A}$ as in the case of single pair FRET-based communication.

In order to demonstrate the dependence of $P_{F R E T, D A}$ on $R_{D R}$ and $R_{R A}$, we theoretically construct a multi-step communication model using the previously mentioned fluorescent proteins in linear arrangement and in a medium of water at $25^{\circ} \mathrm{C}$ assuming rapid randomization of relative orientation of molecules. We select ECFP as the donor, EYFP as the relay and EGFP (Enhanced Green Fluorescent Protein) as the acceptor molecule. The Förster distances between these molecules under given constraints are measured in [22] as $R_{0, E C F P-E Y F P}=4.92 \mathrm{~nm}$ and $R_{0, E Y F P-E G F P}=$ $3.25 \mathrm{~nm}$. The dependence is shown in Fig. 5. As it is seen, in order to optimize this two-step channel for high FRET efficiency and long communication range, it would be a wise decision to set $R_{D R}$ greater than $R_{R A}$, because $R_{0, D R}$ is greater than $R_{0, R A}$.

The idea of multi-step can be applied to realize longer communication with more than one relay in the same manner. For example, in the experimental study [16], a relatively high FRET efficiency of $\sim 68 \%$ is achieved with five flurophores over a distance of $\sim 13 \mathrm{~nm}$. We believe, with the discoveries of high efficiency FRET pairs and further improvements on locating and orienting techniques of fluorophores, nanoscale communication can be realized over longer internodal distances with less relay nodes by using multi-step FRET technique and the advantages of this communication paradigm over wiring the nanomachines together such as mobility and minimizing energy and budget become more clear.

\section{AN INFORMATION THEORETICAL ANALYSIS FOR FRET-BASED COMMUNICATION}

The FRET Channel is modeled similar to Z-channel with On-Off Keying (OOK) Modulation assuming the excitation period i.e., $T_{H}$, is large enough to prevent ISI. Every time when the laser excites the donor at the beginning of the interval $T_{H}$, i.e., it intends to transmit bit 1 with probability $P_{F}$, the probability of FRET occurrence during that interval determines the success of transmission of bit 1. Thus, using (8), TN achieves to deliver bit 1 with probability of $p_{1}$ given in terms of intermolecular distance $R$ as follows

$$
p_{1}[R]=\frac{R_{0}^{6}}{R_{0}^{6}+R^{6}}
$$

Therefore, the probability of failure of transmitting bit 1 when the donor is excited is $\left(1-p_{1}[R]\right)$.

When the donor is not excited at the beginning of an interval, the probability of FRET abstinence during that interval gives the success probability of bit 0 . The only noise source for that transmission might be an external laser source exciting the acceptor molecule and causes the acceptor to fluorescence randomly. In our model with one information source, assuming there is no noise factor that affects the channel and the receiver is reasonable. Hence, the successful transmission probability of bit 0 is unity, i.e., $p_{0}=1$. Therefore, in this case, the failure probability of transmitting bit 0 becomes $\left(1-p_{0}\right)=0$.

Although we disregard the external noise factors, the channel acts like a noisy channel since the probability of FRET occurrence is intrinsically not equal to 1 . According to the transmission probabilities, the transition matrix of the Zchannel considering $X$ as the transmitted bit by TN, and $Y$ as the received bit by $\mathrm{RN}$ is given as

$$
P(Y \mid X)=\left[\begin{array}{cc}
\left(1-P_{F}\right) p_{0} & \left(1-P_{F}\right)\left(1-p_{0}\right) \\
P_{F}\left(1-p_{1}[R]\right) & P_{F} p_{1}[R]
\end{array}\right]
$$

The simplified form for $p_{0}=1$ can be given by

$$
P(Y \mid X)=\left[\begin{array}{cc}
\left(1-P_{F}\right) & 0 \\
P_{F}\left(\frac{R^{6}}{R_{0}^{6}+R^{6}}\right) & P_{F}\left(\frac{R_{0}^{6}}{R_{0}^{6}+R^{6}}\right)
\end{array}\right]
$$

Consequently, the mutual information $I(X ; Y)$ between $X$ and $Y$ can be inferred from the transition matrix as

$$
I(X ; Y)=H\left(P_{F} p_{1}[R]\right)-P_{F} H\left(1-p_{1}[R]\right),
$$

where $H($.$) denotes the binary entropy. Therefore, the capacity$ of the FRET channel, $C_{F}$, can be given by maximizing the mutual information as follows

$$
C_{F}=\max [I(X ; Y)]
$$

It is possible to increase the channel capacity that varies in accordance with some external and intrinsic parameters by selecting appropriate excitation probabilities, i.e., $P_{F}$. 


\section{NumericAl ANALYSIS}

In this section, we present the numerical analysis performed over the mutual information expression given in (14) to show how the FRET-based communication capacity varies according to some environmental parameters and some intrinsic parameters that are specific to the employed FRET pair. The aim of this analysis is to determine the appropriate configuration of FRET-based communication parameters, which can achieve high communication capacity according to changing environmental parameters. We perform the numerical analysis using MATLAB. The simulation parameters can be seen in Table I.

\section{A. Effect of Intermolecular Distance}

For the first analysis, we investigate the effect of the intermolecular distance $(R)$ on the capacity of FRET-based communication channel. The analysis is carried out with single donor and single acceptor configuration using ECFP-EYFP as the FRET pair assembled on the nanomachines assuming rapid randomization of the relative orientation of the molecules as well as the nanomachines in a medium of water at $25{ }^{\circ} \mathrm{C}$.

Selecting the medium and orientation parameters as specified before, the Förster radius for ECFP - EYFP pair is calculated as $R_{0}=4.92 \mathrm{~nm}$ [22]. In Fig. 6-a, mutual information $(I(X ; Y))$ given in (14) is shown with varying excitation probability of the donor $\left(P_{F}\right)$ for different $R$. For $R$ values higher than the $R_{0}$, the probability of FRET in the case of donor excitation, i.e., $p_{1}$, significantly decreases. As a result, the transmission of bit 1 can be erroneous when the distance between TN and RN is large. Therefore, the capacity decreases for higher $R$. On the other hand, when $R$ is less than $R_{0}, p_{1}$ increases. Therefore, the capacity increases with decreasing internodal distance. Consequently, it is necessary to select appropriate $R$ and $P_{F}$ according to the assembled donor - acceptor pair to achieve higher communication capacity. The capacity is maximized for $R=3 \mathrm{~nm}$ by $P_{F}=0.474$. We find $C_{\max }=0.86 \mathrm{bit}$. Hence, we can communicate more information by using input symbol 0 more frequently than 1 with intermolecular distance of $3 \mathrm{~nm}$.

\section{B. Effect of Medium}

In this analysis, we investigate the channel capacity for different media. The analysis is carried out with single ECFPEYFP pair as the donor and the acceptor assembled on TN and $\mathrm{RN}$, respectively. The nanomachines are located in different mediums and separated by a distance of $4 \mathrm{~nm}$ assuming rapid randomization of relative orientation of the molecules.

For ECFP - EYFP pair, the Förster radius calculated in [22] changes in accordance with the refractive index of the medium. In Fig. 6-b, mutual information $I(X ; Y)$ given in (14) is shown for varying excitation probability of the donor $\left(P_{F}\right)$ for different media and different refractive indices. As the refractive index of the medium decreases, the Förster radius given in (1) increases. Therefore, the probability of FRET, i.e., successful transmission probability of bit 1 increases. As a consequence, the capacity of the channel increases with decreasing refractive index. The capacity is maximized for vacuum by $P_{F}=0.43$. We find $C_{\max }=0.57$ bit.
TABLE I

SIMULATION PARAMETERS

\begin{tabular}{|l|l|}
\hline \multirow{3}{*}{ Donor - Acceptor pair } & EBFP - DsRed \\
& ECFP - EYFP \\
& EGFP - EYFP \\
& ECFP - EYFP - EGFP (relayed case) \\
\hline Intermolecular distance $(R)$ & $(3-6) n m$ \\
\hline \multirow{2}{*}{ Refractive index $(n)$} & $1($ vacuum) \\
& 1.3342 (water at $\left.25^{\circ} \mathrm{C}\right)$ \\
\hline \multirow{2}{*}{ Orientation factor $\left(\kappa^{2}\right)$} & 1.5185 (silicon oil at $\left.25^{\circ} \mathrm{C}\right)$ \\
& $2 / 3$ (rapid randomization) \\
& 4 (parallel dipole moments) \\
\hline
\end{tabular}

\section{Effect of Relative Orientation Factor}

Here, we investigate the effect of relative orientation factor $\left(\kappa^{2}\right)$ on the channel capacity using ECFP - EYFP as the donor - acceptor pair assembled on the nanomachines. The nanomachines are located in a medium of water at $25^{\circ} \mathrm{C}$ and separated by a distance of $4 \mathrm{~nm}$.

Relative orientation factor is a measure of the relative orientation of the donor emission dipole moment and the acceptor absorption dipole moment. Determining the exact orientations of donor and acceptor molecules is impossible at this point. However, many of the studies in the literature about FRET assume rapid randomization of the relative orientation of the dipole moments. The orientation factor is $2 / 3$ in the case of rapid randomization. In addition, we investigate the mutual information when the orientation of the dipole moments of the molecules are parallel. In this case, the orientation factor reaches its maximum value, i.e., $\kappa^{2}=4$. The result of the analysis seen in Fig. 6-c reveals that the parallel orientation can significantly increase the capacity of FRET-based channel compared to rapid randomization. When further advances in the nanotechnology make it possible to control the orientation of molecules, orienting the dipole moments of the donor and the acceptor molecules in parallel will be a wise strategy to achieve higher communication capacities. For parallel orientation, the capacity is $P_{F}=0.47$. We find $C_{\max }=0.70 \mathrm{bit}$.

\section{Capacity Analysis for Different FRET Pairs}

In this analysis, we investigate the communication capacity for various donor - acceptor pairs with different spectral properties, assembled on TN and RN respectively. The nanomachines are located in a medium of water at $25^{\circ} \mathrm{C}$ and separated by a distance of $4 \mathrm{~nm}$. The molecules that constitute the FRET pairs are selected among the variants of Green Fluorescent Protein (GFP). The selected donor - acceptor pairs are commonly used in FRET studies and there is a wide variety of studies about GFP variants in the literature.

There is a direct relationship between the spectral overlap of molecules and $R_{0}$ as well as FRET efficiency, i.e., the transmission probability of bit $1\left(p_{1}\right)$. As the overlap of the spectra increases, $R_{0}$ as well as $p_{1}$ increases. For the pair of Enhanced Blue Fluorescent Protein (EBFP) and Red Fluorescent Protein (DsRed), the spectral overlap is the minimum among the selected pairs. Therefore, $R_{0}$ for EBFP and DsRed is the minimum and calculated as $3.17 \mathrm{~nm}$ [22]. Conversely, 


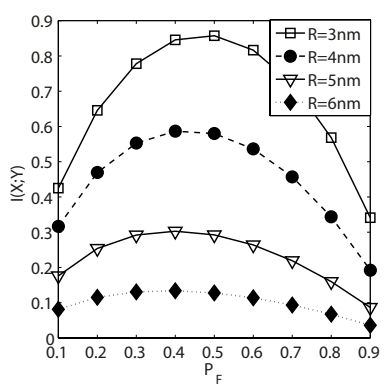

(a) Varying $R$

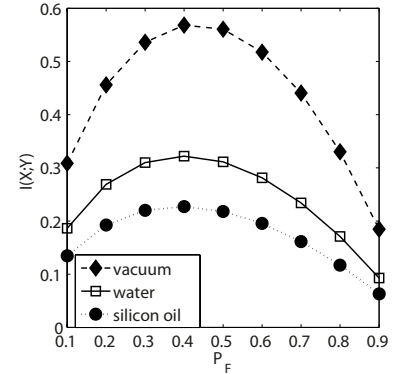

(b) Varying media

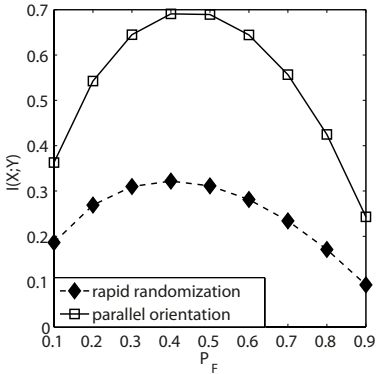

(c) Varying $\kappa^{2}$

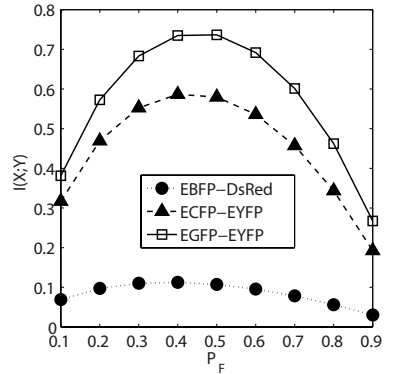

(d) Varying fluorophores

Fig. 6. $I(X ; Y)$ in bits/symbol for several internodal distance $(R)$, medium, relative orientation and FRET-pair selections with varying $P_{F}$.

the overlap between the emission spectrum of Enhanced Green Fluorescent Protein (EGFP) and the absorption spectrum of EYFP is the maximum. As a result, $R_{0}$ for EGFP - EYFP pair is the maximum and calculated as $5.64 \mathrm{~nm}$ [22]. For ECFP - EYFP pair, $R_{0}$ is previously given as $4.92 \mathrm{~nm}$ [22].

Fig. 6-d demonstrates the mutual information, $I(X ; Y)$, given in (14) for varying excitation probabilities $\left(P_{F}\right)$. As expected, the capacity is higher for the pair EGFP - EYFP because of the higher transmission probability of bit $1\left(p_{1}\right)$ as the consequence of higher spectral overlap. As the spectral overlap decreases, the capacity also decreases. Therefore, for the pair EBFP - DsRed, the capacity is the minimum among the others. The selection of the donor and acceptor pair with larger spectral overlap is the key strategy in order to achieve higher communication capacity. The capacity is maximized for EGFP - EYFP by $P_{F}=0.45$. We find $C_{\max }=0.74$ bit.

\section{E. Capacity Analysis for Long-range FRET-based Communi- cation Channel with Multi-step FRET}

Here, we analyze the multi-step FRET-based communication channel information theoretically for the simplest form with only one relay in a similar manner with the single-pair analyses. The main difference of the multi-step case is the determination of $p_{1}$. Assuming that a proper selection for excitation period $\left(T_{H}\right)$ is made, neglecting direct excitation and using the fact that $p_{1}=P_{F R E T}, p_{1}$, i.e., the probability of successful transmission of bit 1 between $\mathrm{TN}$ and $\mathrm{RN}$ for the linear two-step donor-relay-acceptor arrangement can be expressed as the same as the right-hand side of (12) in terms of the intermolecular distances. With the same assumption for $T_{H}$, the probability of success in transmission of bit 0 between $\mathrm{TN}$ and $\mathrm{RN}\left(p_{0, D A}\right)$ is 1 . Therefore, the multi-step channel also shows the Z-channel characteristics.

For a linear arrangement of the previously mentioned triplet ECFP-EYFP-EGFP on TN, HN and RN respectively which are located in a medium of water at $25^{\circ} \mathrm{C}$ and assuming rapid randomization of relative orientation of the molecules on the nanomachines, i.e., $\kappa^{2}=2 / 3$, the dependence of mutual information $(I(X ; Y))$ on $R_{D R}$ and $R_{R A}$ is analyzed using (14) and the results are demonstrated in Fig. 7. As it is seen in Fig. 7, the dependence of the reliability of the channel on internodal distances is in parallel with the results obtained for efficiency dependence in Fig. 5. Particularly, we conclude that using bit 0 more frequently than bit 1 , i.e., decreasing $P_{F}$, results in an increase in the mutual information and the capacity for the same arrangement.

\section{CONCLUSION}

In this study, we propose a novel molecular communication technique exploiting a well-known phenomenon FRET, for the first time in the literature. We define a realistic communication channel model for a single transmitter-receiver pair (point-topoint) within the scope of FRET theory. Succeeding that, the capacity of the newborn channel is formalized informationtheoretically and the variation in the communication channel capacity is analyzed for different environmental and intrinsic parameters. The result of analysis reveals that the capacity of the channel can be increased significantly by appropriately choosing the parameters in accordance with each other.

Throughout the paper, we show that with the relatively low dependency on the environmental factors, high level controllability of the parameters and simplicity, FRET-based molecular communication model stands as a promising solution to high rate nanoscale communication between nanomachines. Furthermore, we show the potential of the model for long range nanonetworks by serially connecting the channels using relay nanomachines. In parallel to the FRET studies in the fluorescent spectroscopy area and with further investigations over this model, broadcast and multiple access methods and more reliable communication as well as new modulation techniques can be realized over this channel.

\section{REFERENCES}

[1] I. F. Akyildiz, F. Brunetti, C. Blazquez, "Nanonetworks: A new communication paradigm," Computer Networks, vol. 52, no. 12, pp. 2260-2279, Aug 2008.

[2] I. F. Akyildiz, M. J. Jornet, "Electromagnetic wireless nanosensor networks," Nano Communication Networks, vol. 1, no. 1, pp. 3-19, March 2010.

[3] T. Nakano, M. Moore, "Molecular Communication Paradigm Overview," Journal of Next Generation Information Technology, vol. 2, pp. 9-16, 2011.

[4] V. V. Didenko, "DNA probes using fluorescence resonance energy transfer (FRET): designs and applications," Biotechniques, vol. 31, no. 5, pp. 1106-1121, Nov 2001.

[5] E. A. Jares-Erijman, T. M. Jovin, "FRET imaging," Nature Biotechnology, vol. 21, pp. 1387-1395, 2003.

[6] L. Stryer, "Fluorescence Energy Transfer as a Spectroscopic Ruler," Annual Review of Biochemistry, vol. 47, pp. 819-846, July 1978.

[7] T. Heyduk, "Measuring protein conformational changes by FRET/LRET," Current Opinion in Biotechnology, vol. 13, no. 4, pp. 292-296, Aug 2002. 


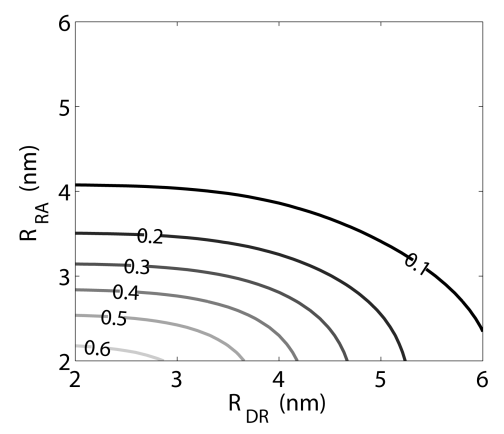

(a) $P_{F}=0.2$

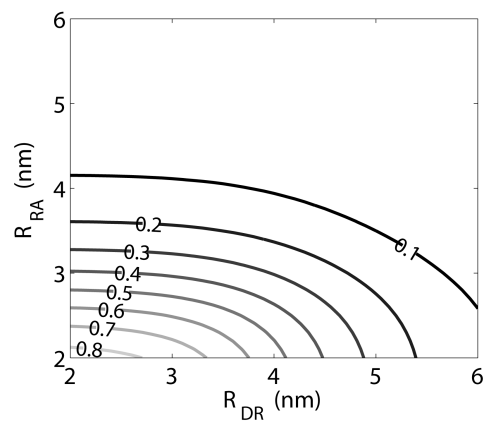

(b) $P_{F}=0.5$

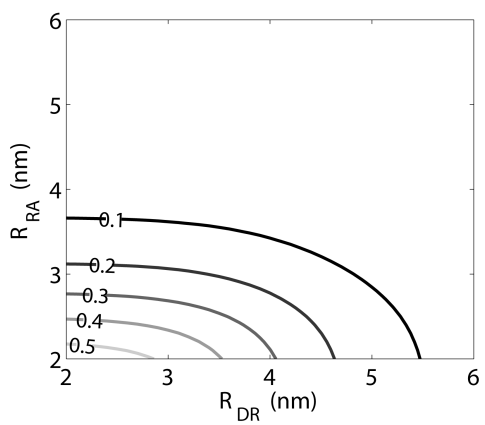

(c) $P_{F}=0.8$

Fig. 7. $I(X ; Y)$ in bits/symbol for several $P_{F}$ with varying $R_{D R}$ and $R_{R A}$ for ECFP(D)-EYFP(R)-EGFP(A) configuration.

[8] S. Lloyd, "A Potentially Realizable Quantum Computer," Science, vol. 261, no. 5128, pp. 1569-1571, Sep 1993.

[9] S. K. Sekatskii, M. Chergui, G. Dietler, "Coherent fluorescence resonance energy transfer: Construction of nonlocal multiparticle entangled states and quantum computing," Europhysics Letters, vol. 63, p. 21, 2003.

[10] T. Nakano, T. Suda, M. Moore, R. Egashira, A. Enomoto, K. Arima, "Molecular communication for nanomachines using intercellular calcium signaling," in Proc. of the 5th IEEE Conf. on Nanotechnology, vol. 2, pp. 478481, Nagoya, Japan, July 2005,.

[11] L. P. Giné, I. F. Akyildiz, "Molecular communication options for long range nanonetworks," Computer Networks, vol. 53, no. 16, pp. 2753-2766, Nov 2009.

[12] M. Gregori, I. F. Akyildiz, "A new nanonetwork architecture using flagellated bacteria and catalytic nanomotors," IEEE J. Sel. Areas Commun., vol. 28, no. 4, pp. 612-619, May 2010.

[13] B. Atakan, O. B. Akan, "Carbon nanotube-based nanoscale ad hoc networks," IEEE Commun. Mag., vol. 48, no. 6, pp. 129-135, June 2010

[14] B. J. MacLennan, "Morphogenesis as a model for nano communication," Nano Communication Networks, vol. 1, no. 3, pp. 199-208, Sep 2010.

[15] M. Kuscu, O. B. Akan, "A nanoscale communication channel with fluorescence resonance energy transfer (FRET)," in Proc. of the 1st IEEE International Workshop on Molecular and Nano Scale Communication (MoNaCom), held in conjunction with IEEE INFOCOM, pp. 425-430, Shanghai, China, April 2011.

[16] M. Heilemann, R. Kasper, P. Tinnefeld, M. Sauer, "Dissecting and Reducing the Heterogeneity of Excited-State Energy Transport in DNABased Photonic Wires," J. Am. Chem. Soc., vol. 128, no. 51, pp. $1686416875,2006$.

[17] H. M. Watrob, C. Pan, M. D. Barkley, "Two-Step FRET as a Structural Tool," J. Am. Chem. Soc., vol. 125, no. 24, pp. 7336-7343, 2003.

[18] J. R. Lakowicz, Principles of Fluorescence Spectroscopy, 3rd ed., Baltimore, MD: Springer, 2006.

[19] T. Förster, "Zwischenmolekulare Energiewanderung und Fluoreszenz," Annalen der Physik, vol. 437, no. 1-2, pp. 55-75, 1948.

[20] R. E. Dale, J. Eisinger, W. E. Blumberg, "The orientational freedom of molecular probes. The orientation factor in intramolecular energy transfer," Biophysical Journal, vol. 26, no. 2, pp. 161-193, May 1979.

[21] H. Chen, H. L. Puhl, S. V. Koushik, S. S. Vogel, S. R. Ikeda, "Measurement of FRET Efficiency and Ratio of Donor to Acceptor Concentration in Living Cells," Biophysical Journal, vol. 91, no. 5, pp. 39-41, Sep 2006.

[22] G. H. Patterson, D. W. Piston, B. G. Barisas, "Förster Distances between Green Fluorescent Protein Pairs," Analytical Biochemistry, vol. 284, no. 2, pp. 438-440, Sep 2000.

[23] B. A. Pollok, R. Heim, "Using GFP in FRET-based applications," Trends in Cell Biology, vol. 9, no. 2, pp. 57-60, Feb 1999.

[24] C. Berney, G. Danuser, "FRET or No FRET: A Quantitative Comparison," Biophysical Journal, vol. 84, no. 6, pp. 3992-4010, June 2003.

[25] R. C. Dunn, "Near-Field Scanning Optical Microscopy," Chemical Reviews, vol. 99, no. 10, pp. 2891-2928, 1999.

[26] R. Pepperkok, A. Squire, S. Geley, P. I. H. Bastiaens, "Simultaneous detection of multiple green fluorescent proteins in live cells by fluorescence lifetime imaging microscopy," Current Biology, vol. 9, no. 5, pp. 269-274, March 1999.

[27] S. Vyawahare, S. Eyal, K. D. Mathews, S. R. Quake, "Nanometer-scale Fluorescence Resonance Optical Waveguides," Nano Letters, vol. 4, no. 6, pp. 1035-1039, 2004.

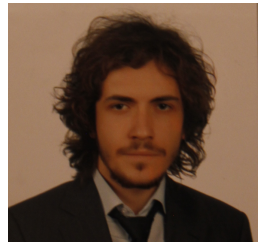

Murat Kuscu (S'11) received his B.Sc. degree in electrical and electronics engineering from Middle East Technical University, Ankara, Turkey, in July 2011. He is currently a research assistant at Nextgeneration and Wireless Communications Laboratory (NWCL) and pursuing his M.Sc. degree in electrical and computer engineering at Koc University, Istanbul, Turkey. His current research interests include nano communications.

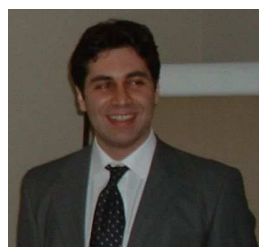

Ozgur B. Akan (M'00-SM'07) received the B.S. and M.S. degrees in electrical and electronics engineering from Bilkent University and Middle East Technical University, Ankara, Turkey, in 1999 and 2001, respectively. He received the Ph.D. degree in electrical and computer engineering from the Broadband and Wireless Networking Laboratory, School of Electrical and Computer Engineering, Georgia Institute of Technology, Atlanta, in 2004. He is currently Associate Professor with the Department of Electrical and Electronics Engineering, Koc University and the Director of Next-generation and Wireless Communications Laboratory (NWCL).His current research interests are in wireless communications, acoustic communications, nano communications, information theory.

Dr. Akan is an Associate Editor for IEEE Transactions on Vehicular Technology, International Journal of Communication Systems (Wiley), European Transactions on Telecommunications, Nano Communication Networks Journal (Elsevier). He served as an Editor for ACM/Springer Wireless Networks (WINET) Journal (2004-2010), as an Area Editor for AD HOC Networks Journal (Elsevier) (2004-2008), as a Guest Editor for several special issues, as the TPC Co-Chair for the 13th ACM International Conference on Modeling, Analysis and Simulation of Wireless and Mobile Systems (ACM MSWiM 2010), the General Co-Chair for The Third International Conference on Bio-Inspired Models of Network, Information, and Computing Systems (ICST/IEEE BIONETICS 2008), the European Vice Chair for The Second International Conference on Nano-Networks (ICST/ACM Nano-Net 2007), an International Vice Chair for IEEE INFOCOM 2006, and in organizing committees and technical program committees of many other international conferences. He is the Vice President for IEEE Communications Society Turkey Section. He is an IEEE Senior Member (Communications Society), and a member of ACM. He is an IEEE Communications Society (COMSOC) Distinguished Lecturer (2011-2012). He received the IEEE COMSOC Outstanding Young Researcher Award for EMEA Region 2010 (as runner-up), the IBM Faculty Award twice in 2010 and 2008, Turkish Academy of Sciences Distinguished Young Scientist Award 2008 (TUBA-GEBIP). 